\title{
An exploration of the ozone dimer potential energy surface
}

Cite as: J. Chem. Phys. 140, 244311 (2014); https://doi.org/10.1063/1.4884962

Submitted: 01 May 2014 . Accepted: 12 June 2014 . Published Online: 27 June 2014

Luis Miguel Azofra, Ibon Alkorta, and Steve Scheiner

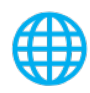

\section{ARTICLES YOU MAY BE INTERESTED IN}

Complexation of $n \mathrm{SO}_{2}$ molecules $(\mathrm{n}=1,2,3)$ with formaldehyde and thioformaldehyde The Journal of Chemical Physics 140, 034302 (2014); https://doi.org/10.1063/1.4861432

Comparison of P...D $(D=P, N)$ with other noncovalent bonds in molecular aggregates The Journal of Chemical Physics 135, 184306 (2011); https://doi.org/10.1063/1.3660355

A new noncovalent force: Comparison of P...N interaction with hydrogen and halogen bonds The Journal of Chemical Physics 134, 094315 (2011); https://doi.org/10.1063/1.3562209

\section{Meet the Next Generation} of Quantum Analyzers And Join the Launch Event on November 17th

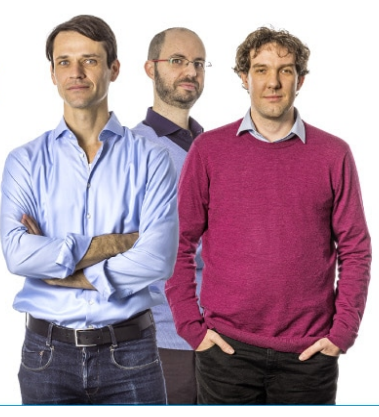




\title{
An exploration of the ozone dimer potential energy surface
}

\author{
Luis Miguel Azofra, ${ }^{1}$ Ibon Alkorta, ${ }^{1}$ and Steve Scheiner ${ }^{2}$,a) \\ ${ }^{1}$ Instituto de Química Médica, CSIC, Juan de la Cierva 3, E-28006 Madrid, Spain \\ ${ }^{2}$ Department of Chemistry and Biochemistry, Utah State University, Logan, Utah 84322-0300, USA
}

(Received 1 May 2014; accepted 12 June 2014; published online 27 June 2014; publisher error corrected 30 June 2014)

\begin{abstract}
The $\left(\mathrm{O}_{3}\right)_{2}$ dimer potential energy surface is thoroughly explored at the ab initio $\operatorname{CCSD}(\mathrm{T})$ computational level. Five minima are characterized with binding energies between 0.35 and $2.24 \mathrm{kcal} / \mathrm{mol}$. The most stable may be characterized as slipped parallel, with the two $\mathrm{O}_{3}$ monomers situated in parallel planes. Partitioning of the interaction energy points to dispersion and exchange as the prime contributors to the stability, with varying contributions from electrostatic energy, which is repulsive in one case. Atoms in Molecules analysis of the wavefunction presents specific $\mathrm{O} \cdots \mathrm{O}$ bonding interactions, whose number is related to the overall stability of each dimer. All internal vibrational frequencies are shifted to the red by dimerization, particularly the antisymmetric stretching mode whose shift is as high as $111 \mathrm{~cm}^{-1}$. In addition to the five minima, 11 higher-order stationary points are identified. () 2014 AIP Publishing LLC. [http://dx.doi.org/10.1063/1.4884962]
\end{abstract}

\section{INTRODUCTION}

The paradigmatic molecule of ozone $\left(\mathrm{O}_{3}\right)$ was the first allotrope of a chemical element to be recognized. Its composition was determined in 1865 by Soret, ${ }^{1}$ and subsequently, in 1867, confirmed by Schönbein. ${ }^{2} \mathrm{O}_{3}$ is found in several atmospheric layers, especially in the stratosphere, where it is most concentrated. It is extremely important due to its ability to capture the most harmful UV radiation via its chemical decomposition to $\mathrm{O}_{2}$, but it has other essential properties associated with environmental issues. ${ }^{3-5}$ The chemistry of $\mathrm{O}_{3}$ is dominated by its high oxidizing power.

Complexes between $\mathrm{O}_{3}$ and various other molecules have been studied computationally. However, while the unique properties of this molecule require high levels of theory, most of the previous work has been carried out at HF, MP2, or DFT (density functional theory) levels, which has limited their reliability. ${ }^{6-12}$ The reproducibility between the theoretical and experimental results through the selection of adequate methodology is crucial in order to offer to the scientific community truly valid results. Otherwise, deficiencies become evident. There have been a small number of studies which employed more appropriate methodologies, e.g., quadratic configuration interaction with single and double excitations (QCISD), coupled-cluster with singles and doubles and linked triples $(\operatorname{CCSD}(\mathrm{T}))$, and/or multi-configurational methods, ${ }^{13,14}$ but most of these have focused on complexes between $\mathrm{O}_{3}$ and $\mathrm{H}_{2} \mathrm{O} .{ }^{15-17}$

Within the experimental context, in their study of the $\mathrm{O} \cdots \mathrm{O}_{3}$ complex through $\mathrm{O}_{3}$ generation with photolysis of an oxygen matrix at $11 \mathrm{~K}$, Schriver-Mazzuoli et al. observed a peak at $\lambda=360 \mathrm{~nm}$ for the photodissociation of the $\left(\mathrm{O}_{3}\right)_{2}$ dimer, but did not provide any structure. ${ }^{18}$ Later, in 2001, Bahou et al. ${ }^{19}$ studied the IR spectroscopy and photochemistry at $266 \mathrm{~nm}$ of $\left(\mathrm{O}_{3}\right)_{2}$ trapped in an argon matrix and con-

\footnotetext{
a) Author to whom correspondence should be addressed. Electronic mail: steve.scheiner@usu.edu.Fax: (+1) 435-797-3390.
}

cluded that this species contains a weak interaction and is not centrosymmetric.

The first structure for the $\left(\mathrm{O}_{3}\right)_{2}$ dimer was proposed by Slanina and Adamowicz, ${ }^{20}$ from a MP2/6-31+G(d) analysis. Their minimum, with $C_{s}$ symmetry, has a dimerization energy of $3.0 \mathrm{kcal} / \mathrm{mol}$. A more recent paper by Gadzhiev et al. ${ }^{21}$ reproduces satisfactorily the experimental behavior of $\mathrm{O}_{3}$ and its homodimer at the CCST(T,full)/cc-pCVTZ level. They identified two minima: one similar to that proposed by Slanina and Adamowicz ${ }^{20}$ and a $C_{i}$ geometry, more stable by $0.25 \mathrm{kcal} / \mathrm{mol}$. Limited primarily to energetics and geometry, neither of these papers provided detailed information about the nature of the weak interactions holding the dimers together.

Interest in the $\left(\mathrm{O}_{3}\right)_{2}$ dimer has been renewed as work progresses into chalcogen bonds, ${ }^{22-28}$ a noncovalent interaction which arises when an atom of that family, e.g., $\mathrm{O}, \mathrm{S}$, or Se, is drawn toward an electron donor site. Electrostatic attractions are typically supplemented by charge transfer from the lone pair(s) of one atom into the $\sigma^{*}$ or $\pi^{*}$ antibonding ${ }^{29-32}$ orbital of the partner molecule. This same idea extends beyond chalcogen atoms, to other electronegative atoms, notably members of the halogen ${ }^{33-38}$ and pnicogen ${ }^{39-44}$ families, and there are very recent works that suggest that even the less electronegative $\mathrm{C}$ group of the periodic table can engage in very similar bonding interactions, known as "tetrel bonds." "45,46

The present work investigates the nature of $\mathrm{O} \cdots \mathrm{O}$ interactions within the context of the ozone dimer. A thorough search of its potential energy surface (PES) reveals five separate minima, each of which is characterized and the nature of its binding analyzed. Other stationary points, including first- and higher-order saddle points are identified, providing a measure of the ability of the various minima to interconvert.

\section{COMPUTATIONAL DETAILS}

The PES of $\left(\mathrm{O}_{3}\right)_{2}$ was explored at the $\operatorname{CCSD}(\mathrm{T})^{47} /$ augcc-pVDZ ${ }^{48}$ computational level. Frequency calculations were 
performed to confirm the nature of the stationary points and to obtain the zero point energy (ZPE). Minima were reoptimized at the CCSD(T)/aug-cc-pVTZ level, to obtain more accurate values. All calculations were performed via the MOLPRO ${ }^{49}$ and Gaussian0 $09^{50}$ packages. Binding energies, $E_{b}$, were computed as the difference in energy between the complex on one hand, and the sum of the energies of the optimized monomers on the other, using the aug-cc-pVDZ and the aug-cc-pVTZ basis sets, and taking into account the ZPE. $E_{b}$ was also extrapolated in the limit of the complete basis set (CBS). ${ }^{51,52}$

The Localized Molecular Orbital Energy Decomposition Analysis method (LMOEDA) ${ }^{53}$ at the CCSD(T) computational level was used to decompose the interaction energy terms via Eq. (1):

$$
E_{\text {int }}=E_{\text {elec }}+E_{\text {exc }}+E_{\text {rep }}+E_{\text {pol }}+E_{\text {disp }} \text {, }
$$

where $E_{\text {elec }}$ is the electrostatic term describing the classical Coulombic interaction of the occupied orbitals of one monomer with those of the other. $E_{\text {exc }}$ and $E_{\text {rep }}$ are the exchange and repulsive component associated with the Pauli exclusion principle, and $E_{p o l}$ and $E_{d i s p}$ correspond to polarization and dispersion terms, respectively. The dispersion energy refers to the $\operatorname{CCSD}(\mathrm{T})$ correction to the Hartree-Fock interaction energy, which contains mainly dispersion and higherorder corrections to the other terms (electrostatic, exchange, repulsion, and polarization). These calculations were carried out with the GAMESS program (version 2013-R1). ${ }^{54}$ The CCSD/aug-cc-pVTZ//CCSD(T)/aug-cc-pVTZ wavefunction was used to analyze the electronic properties of these systems since no $\operatorname{CCSD}(\mathrm{T})$ wavefunction is available within the Gaussian09 program.

Atoms in Molecules (AIM) ${ }^{55}$ theory at the CCSD/aug-ccpVTZ level was applied to analyze these weak interactions, with the aid of the AIMAll program. ${ }^{56}$ The appearance of an AIM bond critical point (BCP) between two centers in the complexes supports the presence of attractive bonding interactions. Numerical integration within the atomic basins was carried out to obtain the atomic charges as well as the atomic contribution to the total energy. The quality of the integration was verified initially with the values of the integrated Laplacian within the atomic basins. In all cases, values smaller than $5 \times 10^{-4}$ a.u. have been obtained. ${ }^{57}$ Thus, the total errors in the charge and energy of the systems, as a sum of the atomic contributions, are smaller than $4 \times 10^{-4} e$ and $0.06 \mathrm{kcal} / \mathrm{mol}$, respectively.

The molecular electrostatic potential (MEP) was analyzed via the WFA-SAS program. ${ }^{58}$ The electron density shift (EDS) maps were calculated as the difference between the electron density of the complex and the sum of those of the monomers in the geometry of the complex using Gaussian09. Finally, the search for stationary points was carried out by the RF method implemented in the MOLPRO package. ${ }^{49}$

\section{RESULTS AND DISCUSSION}

\section{A. $\mathrm{O}_{3}$ monomer}

The isolated ozone molecule is bent, belonging to the $C_{2 v}$ point group. As may be observed in Table I, those computa-
TABLE I. Comparative geometrical (OO distance, in $\AA$, and OOO angle, in $\mathrm{deg})$ and vibrational $\left(\mathrm{A}_{1}\right.$ and $\mathrm{B}_{2}$ modes, in $\left.\mathrm{cm}^{-1}\right)$ variables between experimental and theoretical results in the $\mathrm{O}_{3}$ monomer.

\begin{tabular}{lccccr}
\hline \hline & $d_{\mathrm{OO}}$ & LOOO & $\mathrm{A}_{1}(b)^{\mathrm{a}}$ & $\mathrm{A}_{1}(s t)^{\mathrm{a}}$ & $\mathrm{B}_{2}{ }^{\mathrm{a}}$ \\
\hline Experimental $^{\mathrm{b}}$ & 1.278 & 116.8 & 705 & 1110 & 1042 \\
HF/aug-cc-pVTZ $^{\mathrm{b}}$ & 1.194 & 119.3 & 866 & 1533 & 1404 \\
B3LYP/aug-cc-pVTZ $^{\mathrm{b}}$ & 1.255 & 118.3 & 746 & 1249 & 1189 \\
MP2/aug-cc-pVTZ $^{\mathrm{b}}$ & 1.284 & 116.7 & 741 & 1158 & 2245 \\
QCISD/aug-cc-pVDZ $^{\mathrm{b}}$ & 1.264 & 117.4 & 737 & 1219 & 894 \\
QCISD(T)/aug-cc-pVDZ $^{\mathrm{b}}$ & 1.287 & 116.8 & 695 & 1107 & 895 \\
CCSD/aug-cc-pVDZ $^{\mathrm{b}}$ & 1.259 & 117.3 & 752 & 1248 & 1218 \\
CCSD(T)/aug-cc-pVDZ $^{\mathrm{b}}$ & 1.285 & 116.6 & 703 & 1115 & 971 \\
CCSD(T)/aug-cc-pVTZ & 1.276 & 117.1 & 714 & 1147 & 1039 \\
CASSCF/6-311G(d,p) $^{\mathrm{d}}$ & 1.285 & 116.7 & 707 & 1093 & 1025 \\
\hline \hline
\end{tabular}

$\overline{{ }^{a} \mathrm{~A}_{1}(b) \text { refers to bending, } \mathrm{A}_{1}(s t) \text { to symmetric stretching, and } \mathrm{B}_{2} \text { to anti-symmetric }}$ stretching.

${ }^{b}$ Data obtained from NIST database.

${ }^{\mathrm{c}}$ Calculated in Table I.

${ }^{\mathrm{d}}$ Values from Ref. 13. The active space selection $12 / 9$ for $\mathrm{C}_{2 v}$ ozone monomer was applied.

tional methods that include electron correlation provide acceptable geometry, with $\operatorname{CCSD}(\mathrm{T})$ the most accurate reproduction of experimental quantities. The vibrational frequencies are more sensitive to choice of computational method: HF, DFT, MP2, and CCSD values are significantly in error, in particular the $\mathrm{B}_{2}$ antisymmetric stretching frequency in the last column. The inclusion of triplets in $\operatorname{CCSD}(\mathrm{T})$, along with the aug-cc-pVTZ basis set, is required to achieve acceptable reproduction of all vibrational frequencies.

The MEP on the 0.001 a.u. electron density iso-surface of the ozone molecule contains four negative (red) regions associated with the lone pairs of the terminal atoms as shown in Figure 1(a). Positive (blue) areas are associated with the central atom, with maxima above and below the molecular plane associated with the $\pi$-hole. The values of these maxima are $21.2 \mathrm{kcal} / \mathrm{mol}$, with a much weaker maximum $(\sigma$-hole, $1.3 \mathrm{kcal} / \mathrm{mol}$ ) along the extension of each $\mathrm{O}-\mathrm{O}$ bond. It might be noted that a previous DFT study ${ }^{32}$ overestimated the $\pi$ hole MEP-0.001 a.u. maximum by $27 \%$, another indication of the need for a high level of theory when treating ozone.

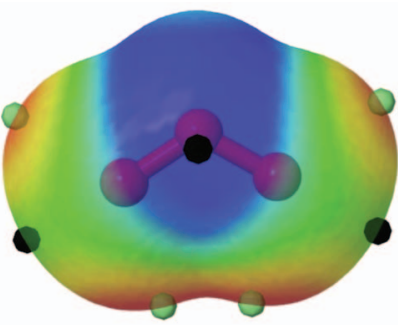

(a)

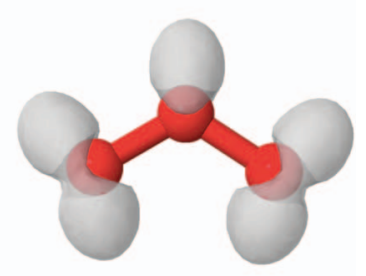

(b)
FIG. 1. (a) MEP on the 0.001 a.u. electron density iso-surface; and (b) ELF (0.8) of the isolated $\mathrm{O}_{3}$ monomer, both calculated at the CCSD/augcc-pVTZ//CCSD(T)/aug-cc-pVTZ computational level. The red and blue regions in the MEP-0.001 a.u. indicate negative and positive regions, respectively, varying between -0.015 and +0.020 a.u. Black and green dots indicate the location of the maxima and minima, respectively, on the molecular surface. 
The electronic localization function (ELF) iso-surface at the CCSD/aug-cc-pVTZ//CCSD(T)/aug-cc-pVTZ level (see Figure 1(b)) shows clearly the location of two basins corresponding to the lone pairs of each of the extreme oxygen atoms that accounts for a population of 2.84 and $2.90 e$ for each atom. In the case of the central oxygen atom, a single basin corresponding to the lone pair electrons is found which integrates to a total of $3.77 \mathrm{e}$. The rest of the electronic population is located in the core basin and the basins associated with the $\mathrm{O}-\mathrm{O}$ bonds.

It is interesting to note that, despite the presence of lone pairs on the central oxygen atom, it is nevertheless surrounded by positive MEP. This observation is confirmed by the integrated electron density within the AIM methodology, which shows negative charge on the terminal atoms $(-0.106 e)$ while the central $\mathrm{O}$ is positive $(0.212 e)$. Thus, the $\mathrm{O}_{3}$ molecule presents a small dipole moment $\left(\mu_{\text {exp }}=0.53 \mathrm{D}^{59}\right.$ and $\mu_{\text {calc }}$ $=0.61 \mathrm{D}$ at the CCSD/aug-cc-pVTZ//CCSD $(\mathrm{T}) /$ aug-ccpVTZ level).

\section{B. Dimers}

\section{Structure and energy}

Five minima were located in the potential energy surface of the ozone dimer. They are illustrated in Figure 2, ordered based upon their binding energy. The latter quantity has been extrapolated to the CBS using the calculated values at the $\operatorname{CCSD}(\mathrm{T}) /$ aug-cc-pVDZ and $\operatorname{CCSD}(\mathrm{T}) /$ aug-cc-pVTZ computational levels (see Table II). Binding energies vary between $-2.24 \mathrm{kcal} / \mathrm{mol}$ for the most stable minimum 1 and $-0.35 \mathrm{kcal} / \mathrm{mol}$ for $\mathbf{5}$. The inclusion of the ZPE correction reduces these energetic values, which then range between -1.68 and $-0.29 \mathrm{kcal} / \mathrm{mol}$. The ZPE corrected and uncorrected binding energies are highly correlated $\left(\mathrm{R}^{2}=0.993\right)$.
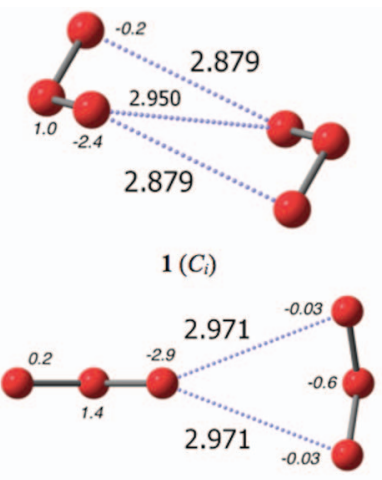

$3\left(C_{s}\right)$
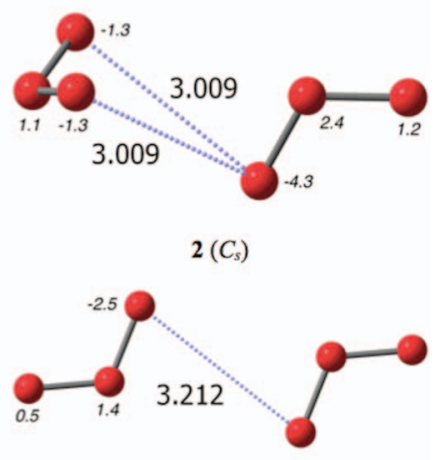

$4\left(C_{2}\right)$

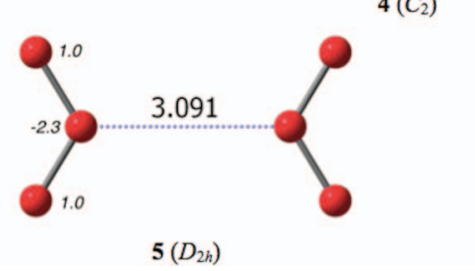

FIG. 2. Structures of the $\left(\mathrm{O}_{3}\right)_{2}$ minima optimized at the CCSD(T)/aug-ccpVTZ level. Broken blue lines link atoms which present interatomic AIM BCPs, with interatomic distances in $\AA$. Complexes are arranged in ascending order of energy. Atomic energy changes upon complexation $(\mathrm{kcal} / \mathrm{mol})$ are displayed in italics. $\mathbf{1}\left(C_{i}\right), \mathbf{2}\left(C_{s}\right), \mathbf{3}\left(C_{s}\right), \mathbf{4}\left(C_{2}\right)$, and $\mathbf{5}\left(D_{2 h}\right)$.
TABLE II. Binding energies, $E_{b}$, computed with aug-cc-pVDZ, aug-ccpVTZ and CBS basis sets, all with $\operatorname{CCSD}(\mathrm{T}) . \Delta H$ and $\Delta G$ are calculated at the CCSD(T)/aug-cc-pVDZ level for $T=298.15 \mathrm{~K}$. All energies in $\mathrm{kcal} / \mathrm{mol}$.

\begin{tabular}{lccccccr}
\hline \hline Dimer & Symmetry & VDZ & VTZ & CBS & CBS+ZPE & $\Delta H$ & $\Delta G$ \\
\hline $\mathbf{1}$ & $C_{i}$ & -2.35 & -2.24 & -2.24 & -1.68 & -1.73 & 7.23 \\
$\mathbf{2}$ & $C_{s}$ & -2.14 & -1.89 & -1.87 & -1.51 & -2.69 & 8.52 \\
$\mathbf{3}$ & $C_{s}$ & -1.52 & -1.36 & -1.28 & -0.98 & -2.10 & 8.77 \\
$\mathbf{4}$ & $C_{2}$ & -1.37 & -1.16 & -1.08 & -0.90 & -2.54 & 9.88 \\
$\mathbf{5}$ & $D_{2 h}$ & -0.87 & -0.50 & -0.35 & -0.29 & -2.67 & 11.24 \\
\hline \hline
\end{tabular}

Distortion energies for the monomers within each dimer are quite small (less than $0.01 \mathrm{kcal} / \mathrm{mol}$ ) consistent with the very weak interactions.

The most stable minimum may be characterized as slipped parallel, with the two $\mathrm{O}_{3}$ molecules lying in parallel planes, with their terminal $\mathrm{O}$ atoms facing one another. The two molecular planes are perpendicular in $\mathbf{2}$ and $\mathbf{3}$, while both molecules lie in the same plane in $\mathbf{4}$ and $\mathbf{5}$. Minimum $\mathbf{1}$ contains the shortest intermolecular O . . O distance of $2.879 \AA$. On the other hand, these intermolecular distances are not well correlated with binding energy. For example, even though the $\mathrm{O}$...O intermolecular distances are shorter in $\mathbf{3}$ than in $\mathbf{2}$, the latter is more stable than the former. Likewise, $\mathbf{5}$ contains a shorter $\mathrm{R}(\mathrm{O} \cdots \mathrm{O})$ than does 4 . One indication of stability is associated with the number of intermolecular bond paths, as analyzed via AIM. The most stable 1 structure contains three such bonds, while there are two bonds in $\mathbf{2}$ and $\mathbf{3}$, and only one in $\mathbf{4}$ and $\mathbf{5}$.

The last two columns of Table II contain $\Delta H$ and $\Delta G$ for the dimerization of each of the five complexes, within the CCSD(T)/aug-cc-pVDZ framework, evaluated at $T$ $=298.15 \mathrm{~K}$. With the exception of dimer $\mathbf{1}, \Delta H$ is more negative than is the binding energy $E_{b}$, albeit by only a small amount. Note that the energetic ordering of the five minima is different for $\Delta H$ than for $E_{b}$. Rather than being most stable, dimer 1 has the least negative value of $\Delta H$, and 2, 4, and 5 are nearly equal in enthalpy. After entropic contributions are added, however, 1 again reclaims its status as most stable. In fact, the free energy ordering is identical to that of $E_{b}$.

In order to gain insight into the source of the interaction energy, various components of the interaction energy were evaluated by the LMOEDA energy decomposition scheme. These quantities are reported in Table III, which shows the repulsion term to be the largest in absolute value for four of the five structures. Of the various attractive terms, exchange is most important, followed by dispersion, electrostatics, and

TABLE III. LMOEDA energy components $(\mathrm{kcal} / \mathrm{mol})$ calculated at CCSD(T)/aug-cc-pVTZ level.

\begin{tabular}{lcccccc}
\hline \hline Dimer & $E_{\text {elec }}$ & $E_{\text {exc }}$ & $E_{\text {rep }}$ & $E_{\text {pol }}$ & $E_{\text {disp }}$ & $E_{\text {int }}$ \\
\hline $\mathbf{1}$ & -1.81 & -4.94 & 8.47 & -0.65 & -3.31 & -2.24 \\
$\mathbf{2}$ & -1.69 & -3.20 & 5.55 & -0.40 & -2.15 & -1.89 \\
$\mathbf{3}$ & -0.83 & -2.51 & 4.34 & -0.37 & -1.98 & -1.36 \\
$\mathbf{4}$ & -1.00 & -1.42 & 2.51 & -0.22 & -1.02 & -1.17 \\
$\mathbf{5}$ & 0.41 & -0.52 & 0.96 & -0.14 & -1.21 & -0.51 \\
\hline \hline
\end{tabular}



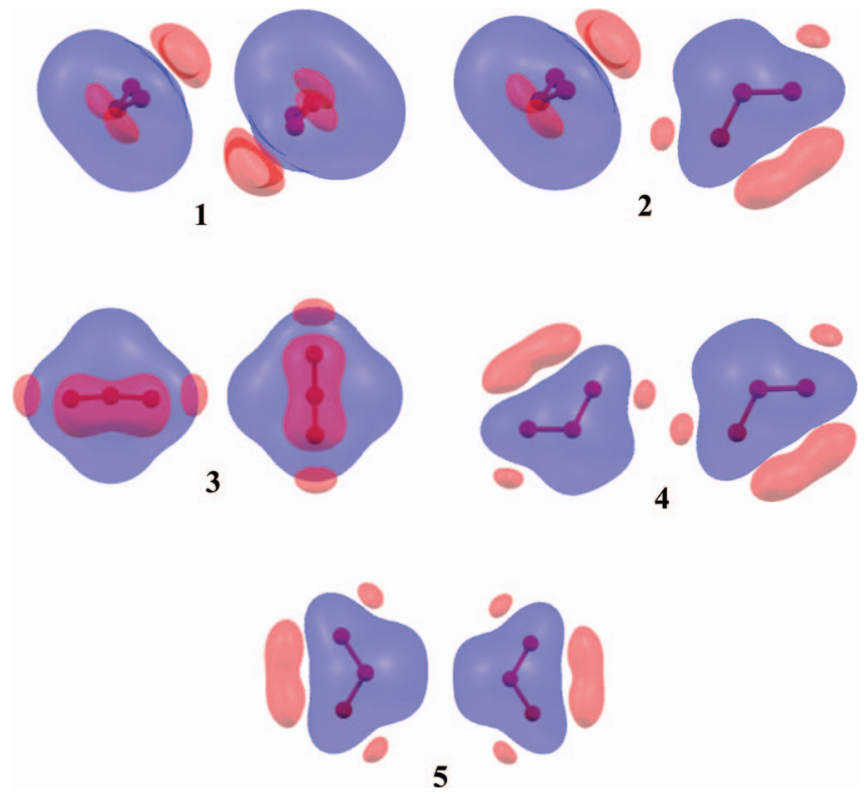

FIG. 3. Mutual orientations of electrostatic potentials of dimers 1-5. The \pm 0.014 a.u. contour is displayed for each with blue corresponding to positive and red to negative.

polarization in that order. The small magnitude of the latter term is verified by only very small values of $E(2)$ when these dimers are subjected to natural bond orbital (NBO) analysis, albeit at the HF level. (As a caveat, it should be stressed that NBO can be calculated only at HF and DFT levels.) A scan of the first column of Table III indicates that there is a Coulombic attraction between the monomers in structures 1-4. The source of this attraction is evident in Figure 3, which stresses the overlap between the positive (blue) regions of one molecule and the negative (red) MEP areas of its partner. The positive value for $E_{\text {elec }}$ for $\mathbf{5}$ in Table III is rooted in the Coulombic repulsion evident in Figure 3. It is likely that this repulsion is partly responsible for the low binding energy of 5.

The atomic energetic changes on going from the isolated monomer to the dimer have been calculated with the AIM method and are included in Figure 2. Variations between -4.3 and $2.4 \mathrm{kcal} / \mathrm{mol}$ are found. The atoms involved in intermolecular bond paths are stabilized by negative values ${ }^{60}$ while those not involved are destabilized. There is one exception: the central atom of the monomer at the right of $\mathbf{3}$ in Figure 2, which is stabilized by $-0.6 \mathrm{kcal} / \mathrm{mol}$ although uninvolved in any intermolecular bond. In complexes 1-3 those atoms involved in two simultaneous interactions are more stabilized than those participating in a single interaction.

\section{Electronic properties}

The most stable minimum, $\mathbf{1}$, shows three intermolecular BCPs, while $\mathbf{2}$ and $\mathbf{3}$ present two, and finally, $\mathbf{4}$ and $\mathbf{5}$ only one. The properties of the BCPs in the dimers are clearly clustered into two groups: (i) those with values of electron density around 0.44 a.u. and negative values of its Laplacian, around -0.090 a.u.; and (ii) those with electron density between 0.046 and 0.075 a.u., but with positive Laplacians, between 0.021 and 0.036 a.u. Due to the high values of the electron density and the negative values for their Laplacians, the first group is associated with the obvious covalent bonds within the $\mathrm{O}_{3}$ monomers. The low values of the electron density and positive Laplacians of group 2 correspond to the weak interactions holding the dimers together. These last noncovalent bonds are represented as broken blue lines in Figure 2.

The symmetry of dimers $\mathbf{1}, \mathbf{4}$, and $\mathbf{5}$, are such that the two $\mathrm{O}_{3}$ molecules are equivalent, and consequently there is no net charge transfer between them. The two monomers are inequivalent in $\mathbf{2}$ and $\mathbf{3}$. Even so, the net transfer is small, $0.002 e$ for 2 and $0.001 e$ for 3. A more detailed threedimensional analysis of charge shifts can be visualized via the difference between the total density of each dimer, and the sum of the isolated monomer densities, positioned as they are within the dimer. These electron density shift (EDS) maps are presented in Figure 4 where regions of increased density are indicated by blue, and loss by yellow. The most strongly bound complex $\mathbf{1}$ displays a net increase of density in the region between the two monomers, as does $\mathbf{5}$. The patterns in $\mathbf{2}, \mathbf{3}$, and $\mathbf{4}$, however, are more characteristic of local charge shifts in that the yellow regions of one molecule are paired with blue areas of its partner. In addition, the atomic charge changes upon complexation calculated within the AIM methodology are displayed in Figure 4.

Complexes 3, 4, and 5 show a general pattern that those atoms directly involved in intermolecular interactions increase their electron density (charge more negative) while those not involved in such interactions become more positive, or remain unchanged. Dimer 2 also follows this behaviour,

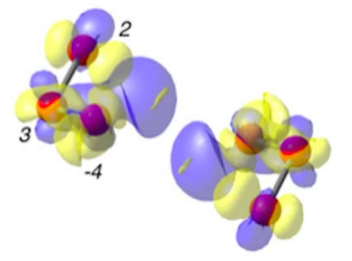

$1\left(C_{i}\right)$
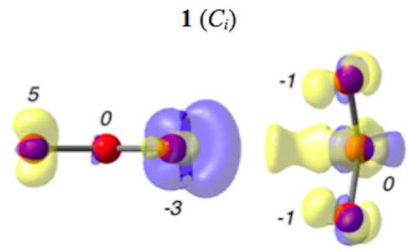

$3\left(C_{s}\right)$

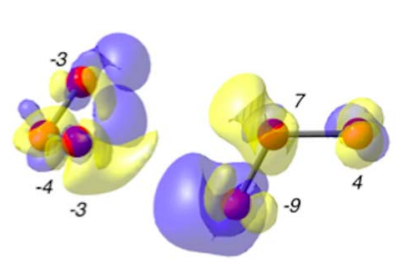

$2\left(C_{s}\right)$

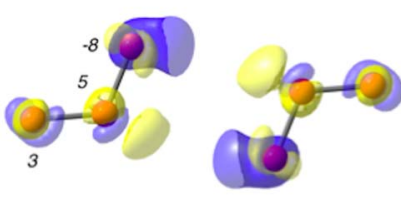

$4\left(C_{2}\right)$
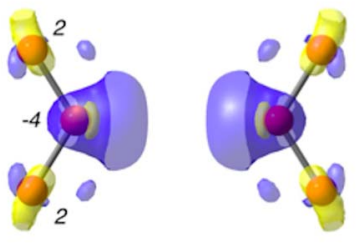

$\mathbf{5}\left(D_{2 h}\right)$

FIG. 4. Electron Density Shifts (EDS) calculated at CCSD/aug-ccpVTZ//CCSD(T)/aug-cc-pVTZ level. Blue and yellow colors refer to gain and loss of density in complex, respectively, relative to isolated monomers. The values of the represented iso-surfaces are \pm 0.00025 a.u. for 1-4 and \pm 0.00010 a.u. for 5. Atomic charge variations upon complexation in me (mili-electrons) at the CCSD/aug-cc-pVTZ//CCSD(T)/aug-cc-pVTZ level are indicated in italics. $\mathbf{1}\left(C_{i}\right), \mathbf{2}\left(C_{s}\right), \mathbf{3}\left(C_{s}\right), \mathbf{4}\left(C_{2}\right)$, and $\mathbf{5}\left(D_{2 h}\right)$ 
TABLE IV. OO bond lengths ( $\AA$ ) and change in vibrational frequencies $\left(\mathrm{cm}^{-1}\right)$ caused by dimerization, calculated at the $\operatorname{CSSD}(\mathrm{T}) /$ aug-cc-pVDZ level.

\begin{tabular}{llrrrc}
\hline \hline System & \multicolumn{1}{c}{$R_{\mathrm{OO}}$} & $\mathrm{A}_{1}(b)^{\mathrm{a}}$ & $\mathrm{A}_{1}(s t)^{\mathrm{a}}$ & $\mathrm{B}_{2}{ }^{\mathrm{a}}$ & Type $^{\mathrm{b}}$ \\
\hline Monomer & 1.285 & 714 & 1147 & 1039 & - \\
$\mathbf{1}$ & $1.286 / 1.284$ & -10 & -32 & -111 & Dissymm. \\
& $1.286 / 1.284$ & -10 & -32 & -49 & Symm. \\
$\mathbf{2}$ & $1.284 / 1.284$ & -9 & -30 & -68 & Dissymm. \\
& $1.286 / 1.283$ & -10 & -31 & -67 & Symm. \\
$\mathbf{3}$ & $1.285 / 1.285$ & -12 & -35 & -76 & Dissymm. \\
& $1.287 / 1.284$ & -10 & -32 & -70 & Symm. \\
$\mathbf{4}$ & $1.287 / 1.284$ & -10 & -32 & -72 & Dissymm. \\
& $1.287 / 1.284$ & -11 & -32 & -66 & Symm. \\
$\mathbf{5}$ & $1.285 / 1.285$ & -15 & -34 & -85 & Dissymm. \\
& $1.285 / 1.285$ & -12 & -33 & -63 & Symm. \\
\hline \hline
\end{tabular}

$\overline{{ }^{a} \mathrm{~A}_{1}(b) \text { refers to bending, } \mathrm{A}_{1}(s t) \text { to symmetric stretching, and } \mathrm{B}_{2} \text { to anti-symmetric }}$ stretching.

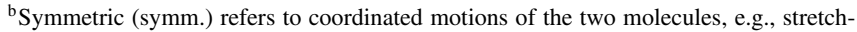
ing of bonds in both molecules simultaneously, and dissymmetric (dissym.) indicates stretching in one molecule coordinated with contractions in the second.

with the exception of a gain of electron density for the central $\mathrm{O}$ atom in the left monomer in Figure 4, even slightly more than the terminal atoms. Finally, complex 1 experiences a gain of electron density only in the terminal $\mathrm{O}$ atoms, those which exhibit two bond paths (see Figure 2).

\section{Spectroscopic properties}

The calculated frequencies of the isolated monomer are compared with the same quantities within the various dimers in Table IV. All frequencies shift to the red. The bending mode is least affected by dimerization, changing by only some $9-15 \mathrm{~cm}^{-1}$. The symmetric stretching frequency red shifts by a nearly uniform amount for all dimers, $30-35 \mathrm{~cm}^{-1}$. The greatest shifts are observed in the antisymmetric stretching, which is as much as $111 \mathrm{~cm}^{-1}$ in $\mathbf{1}$. The other four dimers undergo red shifts of this mode between 63 and $85 \mathrm{~cm}^{-1}$. It is also worth noting that the internal bond lengths in the ozone molecule are changed very little by dimerization, less than $0.002 \AA$.

\section{Other stationary points}

In addition to the minima, the search for stationary points in the potential energy surface of the ozone dimer turned up a group of higher-order saddle points as well. First-, second-, and third-order saddle points are displayed in Figure S1 of the supplementary material, ${ }^{61}$ along with their energies relative to the lowest-energy minimum 1. It is first clear that the various stationary points have energies comparable to the minima themselves. With regard to the first-order saddle points, i.e., transition states, it is not entirely clear which minima they connect. Inspection of the motions of the atoms corresponding to the imaginary frequency of $\mathbf{a}$, for example, appears to connect minimum $\mathbf{1}$ with a symmetric variant of itself, and $\mathbf{b}$ connects to $\mathbf{2}$. The remaining structures are more difficult to assign. Saddle points containing three imaginary fre- quencies (i-k) are symmetric structures. In i $(1.08 \mathrm{kcal} / \mathrm{mol})$ the two monomers are located in perpendicular planes with their dipoles aligned. Terminal $\mathrm{O}$ atoms of the two molecules point directly toward one another in $\mathbf{j}$, whereas the molecular dipoles will repel one another in $\mathbf{k}$. The atomic motions associated with each imaginary frequency are illustrated in Fig. S2 of the supplementary material. ${ }^{61}$

\section{SUMMARY}

There are five minima on the potential energy surface of the ozone dimer. In the most stable of these, the planes of the two molecules are parallel, with some horizontal displacement. The most accurate assessment of its binding energy is $2.24 \mathrm{kcal} / \mathrm{mol}$. Besides exchange energy, the strongest component of the binding is dispersion, followed by a Coulombic attraction. AIM analysis of the wavefunction provides three intermolecular bond paths, all involving the terminal $\mathrm{O}$ atoms. A second minimum is only slightly less stable, with a binding energy of $1.89 \mathrm{kcal} / \mathrm{mol}$. The planes of the two monomers are perpendicular to one another in this structure, and there are two AIM bond paths, again between terminal $\mathrm{O}$ atoms. The remaining three minima are bound by 0.35 to $1.36 \mathrm{kcal} / \mathrm{mol}$, but in all five cases it is dispersion and exchange that are primarily responsible for the attractive force, with a smaller but non-negligible contribution from electrostatics. The only exception is the least stable dimer in which a Coulombic repulsion must be overcome by the other forces.

Interoxygen distances vary from $2.879 \AA$ for the most stable minimum, to as long as $3.212 \AA$. In keeping with the small induction energies, shifts in electron density associated with the formation of the dimers are also small. The formation of any of these five dimers is accompanied by a red shift of all three internal vibrational modes, in particular the asymmetric stretching which is shifted to lower frequency by as much as $111 \mathrm{~cm}^{-1}$. Besides the five minima, 11 different saddle points of varying order were located on the potential energy surface. The energies of these structures are in the same range as those of the minima, which is suggestive of a flat potential energy surface, with an ease of conversion from one minimum to another.

\section{ACKNOWLEDGMENTS}

This work has been supported by NSF-CHE-1026826 and CTQ2012-35513-C02-02 (MINECO) Projects. Also, L.M.A. thanks the MICINN for a Ph.D. grant (Grant No. BES-2010-031225). Computer, storage and other resources from the Division of Research Computing in the Office of Research and Graduate Studies at Utah State University and the CTI (CSIC) are gratefully acknowledged.

${ }^{1}$ J.-L. Soret, C. R. Hebd. Seances Acad. Sci., Ser. C 61, 941 (1865).

${ }^{2}$ M. B. Rubin, Bull. Hist. Chem. 26, 40 (2001).

${ }^{3}$ J. P. D. Abbatt and M. J. Molina, Annu. Rev. Energy Env. 18, 1 (1993). ${ }^{4}$ S. Solomon, Rev. Geophys. 37, 275, doi:10.1029/1999RG900008 (1999).

${ }^{5}$ M. Norval, R. M. Lucas, A. P. Cullen, F. R. de Gruijl, J. Longstreth, Y. Takizawa, and J. C. van der Leun, Photochem. Photobiol. Sci. 10, 199 (2011).

${ }^{6}$ L.-C. Yang and D.-C. Fang, J. Mol. Struct.: THEOCHEM 671, 141 (2004) 
${ }^{7}$ H. Tachikawa and S. Abe, Inorg. Chim. Acta 358, 288 (2005).

${ }^{8}$ B. Flemmig, P. T. Wolczanski, and R. Hoffmann, J. Am. Chem. Soc. 127, 1278 (2005)

${ }^{9}$ S.-d. Jiang, Z.-h. Wang, J.-h. Zhou, Z.-c. Wen, and K.-f. Cen, J. Zhejiang Univ. Sci. A 10, 1327 (2009).

${ }^{10}$ O. A. Loboda and V. V. Goncharuk, J. Water Chem. Technol. 31, 213 (2009).

${ }^{11}$ M. Solimannejad, I. Alkorta, and J. Elguero, Chem. Phys. Lett. 474, 253 (2009).

${ }^{12}$ M. Solimannejad, Mol. Simul. 37, 1071 (2011).

${ }^{13}$ M. Alcamí, I. L. Cooper, O. Mó, and M. Yáñez, J. Chem. Phys. 103, 253 (1995).

${ }^{14}$ A. Mansergas and J. M. Anglada, J. Phys. Chem. A 111, 976 (2007).

${ }^{15}$ H. Tachikawa and S. Abe, Inorg. Chem. 42, 2188 (2003).

${ }^{16}$ P. Kumar and N. Sathyamurthy, Chem. Phys. 415, 214 (2013).

${ }^{17}$ J. M. Anglada, G. J. Hoffman, L. V. Slipchenko, M. M. Costa, M. F. RuizLópez, and J. S. Francisco, J. Phys. Chem. A 117, 10381 (2013).

${ }^{18}$ L. Schriver-Mazzuoli, A. de Saxcé, C. Lugez, C. Camy-Peyret, and A. Schriver, J. Chem. Phys. 102, 690 (1995).

${ }^{19}$ M. Bahou, L. Schriver-Mazzuoli, and A. Schriver, J. Chem. Phys. 114, 4045 (2001).

${ }^{20}$ Z. Slanina and L. Adamowicz, J. Atmos. Chem. 16, 41 (1993).

${ }^{21}$ O. B. Gadzhiev, S. K. Ignatov, M. Y. Kulikov, A. M. Feigin, A. G. Razuvaev, P. G. Sennikov, and O. Schrems, J. Chem. Theory Comput. 9, 247 (2013).

${ }^{22}$ R. E. Rosenfield, R. Parthasarathy, and J. D. Dunitz, J. Am. Chem. Soc. 99, 4860 (1977).

${ }^{23}$ F. T. Burling and B. M. Goldstein, J. Am. Chem. Soc. 114, 2313 (1992).

${ }^{24}$ D. B. Werz, R. Gleiter, and F. Rominger, J. Am. Chem. Soc. 124, 10638 (2002).

${ }^{25}$ M. Iwaoka, S. Takemoto, and S. Tomoda, J. Am. Chem. Soc. 124, 10613 (2002)

${ }^{26}$ C. Bleiholder, D. B. Werz, H. Köppel, and R. Gleiter, J. Am. Chem. Soc. 128, 2666 (2006).

${ }^{27}$ G. Sánchez-Sanz, I. Alkorta, and J. Elguero, Mol. Phys. 109, 2543 (2011).

${ }^{28}$ M. a. Jabłoński, J. Phys. Chem. A 116, 3753 (2012).

${ }^{29}$ J. T. Goettel, P. Chaudhary, P. Hazendonk, H. P. A. Mercier, and M. Gerken, Chem. Commun. 48, 9120 (2012).

${ }^{30}$ L. M. Azofra and S. Scheiner, J. Chem. Phys. 140, 034302 (2014).

${ }^{31}$ L. M. Azofra and S. Scheiner, Phys. Chem. Chem. Phys. 16, 5142 (2014).

${ }^{32}$ J. Murray, P. Lane, T. Clark, K. Riley, and P. Politzer, J. Mol. Model. 18, 541 (2012).

${ }^{33}$ J. P. M. Lommerse, A. J. Stone, R. Taylor, and F. H. Allen, J. Am. Chem. Soc. 118, 3108 (1996)

${ }^{34}$ P. Metrangolo and G. Resnati, Science 321, 918 (2008).

${ }^{35}$ P. Politzer, J. Murray, and M. Concha, J. Mol. Model. 14, 659 (2008).
${ }^{36} \mathrm{P}$. Hobza and K. Müller-Dethlefs, Non-Covalent Interactions (The Royal Society of Chemistry, Cambridge, UK, 2009).

${ }^{37}$ W. Zierkiewicz, D. Michalska, and T. Zeegers-Huyskens, Phys. Chem. Chem. Phys. 12, 13681 (2010).

${ }^{38}$ U. Adhikari and S. Scheiner, Chem. Phys. Lett. 532, 31 (2012).

${ }^{39}$ S. Zahn, R. Frank, E. Hey-Hawkins, and B. Kirchner, Chem. Eur. J. 17, 6034 (2011).

${ }^{40}$ U. Adhikari and S. Scheiner, Chem. Phys. Lett. 536, 30 (2012).

${ }^{41}$ S. Tschirschwitz, P. Lonnecke, and E. Hey-Hawkins, Dalton Trans. 2007, 1377.

${ }^{42}$ S. Scheiner, J. Phys. Chem. A 115, 11202 (2011).

${ }^{43}$ M. Bühl, P. Kilian, and J. D. Woollins, ChemPhysChem 12, 2405 (2011).

${ }^{44}$ S. Scheiner, Acc. Chem. Res. 46, 280 (2013).

${ }^{45}$ A. Bauzá, T. J. Mooibroek, and A. Frontera, Angew. Chem. Int. Ed. 52, 12317 (2013).

${ }^{46}$ S. J. Grabowski, Phys. Chem. Chem. Phys. 16, 1824 (2014).

${ }^{47}$ P. J. Knowles, C. Hampel, and H.-J. Werner, J. Chem. Phys. 99, 5219 (1993).

${ }^{48}$ T. H. J. Dunning, J. Chem. Phys. 90, 1007 (1989).

${ }^{49}$ H.-J. Werner, P. J. Knowles, G. Knizia, F. R. Manby, M. Schütz et al., MOLPRO, version 2012.1, a package of ab initio programs, 2012, see http://www.molpro.net.

${ }^{50}$ M. J. Frisch, G. W. Trucks, H. B. Schlegel et al., GAUSSIAN09, Revision D.01, Gaussian, Inc., Wallingford, CT, 2009.

${ }^{51}$ D. G. Truhlar, Chem. Phys. Lett. 294, 45 (1998)

${ }^{52}$ S. Scheiner, Comp. Theor. Chem. 998, 9 (2012).

${ }^{53}$ P. Su and H. Li, J. Chem. Phys. 131, 014102 (2009).

${ }^{54}$ M. W. Schmidt, K. K. Baldridge, J. A. Boatz, S. T. Elbert, M. S. Gordon, J. H. Jensen, S. Koseki, N. Matsunaga, K. A. Nguyen, S. Su, T. L. Windus, M. Dupuis, and J. A. Montgomery, J. Comput. Chem. 14, 1347 (1993).

${ }^{55}$ R. F. W. Bader, Atoms in Molecules: A Quantum Theory (Clarendon Press, Oxford, UK, 1990).

${ }^{56}$ T. A. Keith, AIMAll, Version 13.11.04, Overland Park, KS, USA, 2013.

${ }^{57}$ I. Alkorta and O. Picazo, Arkivoc 2005, 305 (2005).

${ }^{58}$ F. Bulat, A. Toro-Labbé, T. Brinck, J. Murray, and P. Politzer, J. Mol. Model. 16, 1679 (2010)

${ }^{59}$ R. D. J. Nelson, R. L. J. David, and A. A. Maryott, Selected Values of Electric Dipole Moments for Molecules in the Gas Phase (National Standard Reference Data System, Washington D.C., USA, 1967).

${ }^{60}$ C. F. Matta, J. Hernández-Trujillo, T.-H. Tang, and R. F. W. Bader, Chem. Eur. J. 9, 1940 (2003).

${ }^{61}$ See supplementary material at http://dx.doi.org/10.1063/1.4884962 for Tables S1-S2 with a complete annex containing the Cartesian coordinates for $\mathrm{O}_{3}$ monomer, $\left(\mathrm{O}_{3}\right)_{2}$ minima, and TSs. Also, AIM molecular graphs for $\left(\mathrm{O}_{3}\right)_{2}$ dimers can be found. Also, Figures S1 and S2 contain minima and saddle points and the imaginary frequency motions for the TSs. 